\title{
Decision Fusion in Kernel-Induced Spaces for Hyperspectral Image Classification
}

\author{
Wei Li, Member, IEEE, Saurabh Prasad, Member, IEEE, and James E. Fowler, Senior Member, IEEE
}

\begin{abstract}
The one-against-one (OAO) strategy is commonly employed with classifiers-such as support vector machines-which inherently provide binary two-class classification in order to handle multiple classes. This OAO strategy is introduced for the classification of hyperspectral imagery using discriminant analysis within kernel-induced feature spaces, producing a pair of algorithmskernel discriminant analysis and kernel local Fisher discriminant analysis-for dimensionality reduction, which are followed by a quadratic Gaussian maximum-likelihood-estimation classifier. In the proposed approach, a multiclass problem is broken down into all possible binary classifiers, and various decision-fusion rules are considered for merging results from this classifier ensemble. Experimental results using several hyperspectral data sets demonstrate the benefits of the proposed approach-in addition to improved classification performance, the resulting classifier framework requires reduced memory for estimating kernel matrices.
\end{abstract}

Index Terms-Decision fusion, hyperspectral data, kernel methods, one-against-one (OAO) algorithm.

\section{INTRODUCTION}

$\mathbf{L}$ INEAR discriminant analysis (LDA) (e.g., [1]) is a popular method for supervised feature extraction and dimensionality reduction for hyperspectral imagery (HSI). LDA is a linear transformation that maximizes the between-class scatter while minimizing the within-class scatter in order to provide maximum class separability in the projected subspace. One drawback of LDA for HSI is the inability to capture data structures that are deviated strongly from normality. Accordingly, kernels, which can extract nonlinear discriminant features, have recently become popular for HSI classification tasks. The central idea behind kernel-based methods is to map the data

Manuscript received June 20, 2012; revised November 2, 2012, March 25, 2013, and June 28, 2013; accepted July 2, 2013. This work was supported in part by the National Aeronautics and Space Administration under Grant NNX12AL49G, the University of Houston Startup Funding, and the National Science Foundation under Grant CCF-0915307.

W. Li is with the College of Information Science and Technology, Beijing University of Chemical Technology, Beijing 100029, China (e-mail: liwei089@ ieee.org).

S. Prasad is with the Department of Electrical and Computer Engineering, University of Houston, Houston, TX 77204-4005 USA (e-mail: saurabh. prasad@ieee.org).

J. E. Fowler is with the Department of Electrical and Computer Engineering, Mississippi State University, Mississippi State, MS 39762 USA (e-mail: fowler@ece.msstate.edu).

Color versions of one or more of the figures in this paper are available online at http://ieeexplore.ieee.org.

Digital Object Identifier 10.1109/TGRS.2013.2272760 from the original input space into a kernel-induced space where features are extracted.

In remote sensing, a variety of kernel-projection methods, such as kernel discriminant analysis (KDA) [2], [3] and kernel principal component analysis, have been studied for patternclassification and anomaly-detection tasks [4], [5]. For example, Prasad and Bruce [4] demonstrated that KDA provides superior classification performance as compared to typical LDA when data distributions are such that the resulting decision boundaries are highly nonlinear. Additionally, in [6], kernel local Fisher discriminant analysis (KLFDA) was studied for hyperspectral data analysis. KLFDA bears a similar formulation to KDA with an important difference-it imposes an additional constraint on the mapping that ensures that neighboring points in the input space stay close-by in the projected space and vice versa. Experimental results in [6] with a variety of hyperspectral classification tasks confirm that kernel-based methods such as KDA and KLFDA are powerful feature-extraction techniques for HSI. Note that the LDA and other feature-extraction methods are often used as classifiers themselves by thresholding of the discriminant features [7], [8].

In terms of classification within the resulting kernel-induced spaces, conventional support vector machines (SVMs) [9] are widely used but are essentially binary classifiers. SVMs seek to separate two-class data by learning an optimal decision hyperplane which best separates the training samples in a kernel-induced feature space and to define the model for the classification task by exploiting the concept of margin maximization [10]. Various approaches exist to extend binary classifiers to tasks involving more than two classes [9], [11]. The most popular strategies are the one-against-all (OAA) and one-against-one $(\mathrm{OAO})$ strategies which both enable the use of binary SVMs for multiclass classification [12], [13]. In the OAA strategy, the multiclass classification problem is posed as a series of two-class problems involving one class against all the others combined. It is worth mentioning that, for the OAA strategy, the complexity of the discriminant function between one information class and all the others often increases. On the other hand, in the OAO strategy, each SVM carries out a binary classification between all combinations of information classes, while a decision-fusion rule [14] - typically majority voting (MV) - is employed to assign a final class label to the test sample. Recent work [9], [15], [16] has shown that SVMs are effective for HSI classification, particularly when the size of the training data set is small. We note also that relevance vector machines (RVMs) [17], which incorporate a probabilistic framework, have also been studied with a simple 
Gaussian kernel for hyperspectral classification [18] as well as for OAO classification with band-selection dimensionality reduction [19].

Previous kernel-based discriminant-analysis algorithms for HSI classification learn a single linear transformation in the kernel-induced space [3]. Specifically, they seek to find the linear discriminant in a space obtained via a nonlinear mapping from the input space. However, in multiclass classification tasks, the boundary may still be nonlinear in the kernelinduced space where the data are potentially inseparable - this is especially common when some classes are more difficult to discriminate than others. Such a nonlinear boundary can be approximated by several linear boundaries.

In this paper, in order to overcome the drawback of using a single global transformation for the multiclass task, we propose an implementation of the OAO strategy (commonly used in SVMs) within a kernel-based discriminant-analysis framework (e.g., KDA or KLFDA). Thus, for a $C$-class problem, $C(C-$ 1)/2 KDA-OAO or KLFDA-OAO classifiers are involved. A decision-fusion rule is employed to merge the classification results from these classifiers into the final decision. In this paper, the decision fusion can occur as either "hard" fusion (typically an MV rule) at the class-label level or "soft" fusion at the posterior-probability level which is estimated by a maximum likelihood estimation (MLE) [1] classifier. The classifier, which follows a kernel-based discriminant transformation, usually employs an MLE classifier since the data distribution in kernelprojected spaces tends to be Gaussian [20].

In practical situations, the limited number of available training samples is often insufficient to estimate statistical models for each class because of the high dimensionality of hyperspectral data. One solution for this issue is to extract features into a lower dimensional subspace. In this paper, discriminant features are extracted from a kernel-induced space, and the proposed multiple-classifier system alleviates the small-trainingsample-size problem commonly encountered in hyperspectral classification applications. Real hyperspectral data sets are used for measuring the effectiveness of the proposed classification framework. Experimental results indicate that this system has the following benefits for the proposed KDA-OAO and KLFDA-OAO approaches: 1) Kernel projections, such as KDA and KLFDA, are better able to capture nonlinear discriminant information as compared to linear projections; 2) since each classifier in the proposed system is a binary kernel discriminantanalysis system, the discriminant complexity is reduced from the case in which all classes are treated together; 3) the cost (computational memory or processing time) of the training process is dramatically reduced; 4) fewer training samples are required for optimizing and training the system; and 5) the proposed method is effective even in the presence of noise.

The rest of this paper is organized as follows. In Section II, we provide an introduction to kernel-based discriminant methods, particularly KDA and KLFDA. In Section III, we provide a detailed description of the proposed OAO strategy as applied to KDA and KLFDA as well as the fusion strategy. In Section IV, we describe the experimental hyperspectral data as well as the experimental setup. We conclude by summarizing our results in Section V.

\section{Kernel-Based Discriminant Methods}

\section{A. KDA}

Before describing the proposed KDA-OAO and KLFDAOAO algorithms, we briefly review KDA and KLFDA. KDA seeks to find a projection $\mathbf{w}$ of vectors in a kernel-induced (higher-dimensional) space such that it maximizes Fisher's ratio [1] in that space. For a given nonlinear mapping function $\Phi$, Fisher's ratio in the resulting kernel-induced space can be expressed as

$$
J_{1}(\mathbf{w})=\frac{\left|\mathbf{w}^{\top} S_{\mathbf{b}}^{\Phi} \mathbf{w}\right|}{\left|\mathbf{w}^{\top} S_{\mathbf{w}}^{\Phi} \mathbf{w}\right|}
$$

where $S_{\mathrm{b}}^{\Phi}$ is the between-class scatter matrix and $S_{\mathrm{w}}^{\Phi}$ is the within-class scatter matrix in the space induced by the mapping function $\Phi$.

The benefit of a KDA transformation is that the mapping onto the kernel-induced space provides a linear class-separation structure. For a variety of practical scenarios wherein classconditional distributions are not linearly separable in the input space, KDA ensures much better classification performance than linear dimensionality-reduction techniques [4], [21]. It is interesting to note that the Fisher's discriminant for two classes is equivalent to a least squares regression with the class labels [3]. KDA, when combined with a Mahalanobis minimum-distance metric, can be viewed as a classifier [8]; however, in this paper, KDA is considered to be a supervised dimensionality-reduction method, which is then followed by the MLE classifier.

\section{B. $K L F D A$}

In [6], KLFDA combined with an MLE classifier was studied for remote-sensing applications. KLFDA can be viewed as a kernel extension of local Fisher discriminant analysis (LFDA) [22], which combines the advantages of LDA and localitypreserving projection (LPP) [23]. LDA seeks to find a linear transformation such that the within-class scatter is minimized while the between-class scatter is maximized. Similarly, LPP is a linear manifold-learning technique that seeks to find a map which preserves the local structure of neighboring samples in the input space.

Before introducing KLFDA, a brief introduction to LFDA is provided here. Consider a data set with training samples $\mathbf{X}=$ $\left\{\mathbf{x}_{i}\right\}_{i=1}^{n}$ in $\mathbb{R}^{d}(d$-dimensional feature space) and class label for each sample $y_{i} \in\{1,2, \ldots, C\}$, where $C$ is the number of classes and $n$ is the total number of training samples. Let $n_{l}$ be the number of available training samples for the lth class, $\sum_{l=1}^{C} n_{l}=n$. Define $A_{i, j} \in[0,1]$ as the affinity between $\mathbf{x}_{i}$ and $\mathbf{x}_{j}$

$$
A_{i, j}=\exp \left(-\frac{\left\|\mathbf{x}_{i}-\mathbf{x}_{j}\right\|^{2}}{\gamma_{i} \gamma_{j}}\right)
$$

where $\gamma_{i}=\left\|\mathbf{x}_{i}-\mathbf{x}_{i}^{\left(k^{\prime}\right)}\right\|$ denotes the local scaling of data samples in the neighborhood of $\mathbf{x}_{i}$ and $\mathbf{x}_{i}^{\left(k^{\prime}\right)}$ is the $k^{\prime}$-nearest neighbor of $\mathbf{x}_{i} . A_{i, j}$ is then a symmetric matrix (referred to as 
the affinity matrix) of size $n \times n$ which measures the distance among data samples. In LFDA, the local between-class $S^{(\mathrm{lb})}$ and within-class $S^{(\mathrm{lw})}$ scatter matrices are defined as

$$
\begin{aligned}
S^{(\mathrm{lb})} & =\frac{1}{2} \sum_{i, j=1}^{n} W_{i, j}^{(\mathrm{lb})}\left(\mathbf{x}_{i}-\mathbf{x}_{j}\right)\left(\mathbf{x}_{i}-\mathbf{x}_{j}\right)^{\top} \\
S^{(\mathrm{lw})} & =\frac{1}{2} \sum_{i, j=1}^{n} W_{i, j}^{(\mathrm{lw})}\left(\mathbf{x}_{i}-\mathbf{x}_{j}\right)\left(\mathbf{x}_{i}-\mathbf{x}_{j}\right)^{\top}
\end{aligned}
$$

where $W^{(\mathrm{lb})}$ and $W^{(\mathrm{lw})}$ are $n \times n$ matrices defined as

$$
\begin{gathered}
W_{i, j}^{(\mathrm{lb})}= \begin{cases}A_{i, j}\left(1 / n-1 / n_{l}\right), & \text { if } y_{i}=y_{j}=l \\
\frac{1}{n}, & \text { if } y_{i} \neq y_{j}\end{cases} \\
W_{i, j}^{(\mathrm{lw})}= \begin{cases}\frac{A_{i, j}}{n_{l}}, & \text { if } y_{i}=y_{j}=l \\
0, & \text { if } y_{i} \neq y_{j} .\end{cases}
\end{gathered}
$$

The LFDA transformation is calculated by maximizing Fisher's ratio [1] as defined using these local scatter matrices. From the aforementioned analysis, it is clear that LFDA can obtain good between-class separation in the projection while preserving the within-class local structure at the same time. More details about LFDA can be found in [22].

KLFDA is a kernel extension of LFDA via the kernel trick [15]. In this paper, the kernel function employed is the radial basis function (RBF) [3] expressed as

$$
K\left(\mathbf{x}_{i}, \mathbf{x}_{j}\right)=\exp \left(-\frac{\left\|\mathbf{x}_{i}-\mathbf{x}_{j}\right\|^{2}}{2 \sigma^{2}}\right)
$$

where $\sigma$ is a user-defined parameter of the kernel. For KLFDA, the local within- and between-class scatter matrices are defined in the kernel-induced space. Projection $\tilde{\mathbf{w}}$ in the kernel space that maximizes the modified Fisher ratio is given by the solution of the generalized eigenvalue problem

$$
K L^{(\mathrm{lb})} K \tilde{\mathbf{w}}=\tilde{\Lambda}\left(K L^{(\mathrm{lw})} K+\varepsilon I_{n}\right) \tilde{\mathbf{w}}
$$

where $\tilde{\Lambda}$ is the diagonal eigenvalue matrix; $\varepsilon$ is a small constant; $\tilde{\mathbf{w}}$ is the eigenvector matrix; $K$ is the kernel matrix defined using (7); $L^{(\mathrm{lw})}=D^{(\mathrm{lw})}-W^{(\mathrm{lw})}$, where $D^{(\mathrm{lw})}$ is a diagonal matrix with the $i$ th diagonal element being $D_{i i}^{(\mathrm{lw})}=$ $\sum_{j=1}^{n} W_{i, j}^{(\mathrm{lw})}$; and $L^{(\mathrm{lb})}=L^{(\mathrm{m})}-L^{(\mathrm{lw})}$, where $L^{(\mathrm{m})}$ is the local mixture matrix defined as $L^{(\mathrm{m})}=D^{(\mathrm{m})}-W^{(\mathrm{m})}$ and $D^{(\mathrm{m})}$ is a diagonal matrix with the $i$ th diagonal element being $D_{i i}^{(\mathrm{m})}=\sum_{j=1}^{n} W_{i, j}^{(\mathrm{m})}$.

In KLFDA, the affinity matrix is employed to weight the within-class scatter matrix in the kernel-induced space such that the local neighborhood relationship is preserved. In [6], we demonstrated that KLFDA achieves superior dimensionalityreduction performance as compared to KDA, particularly insofar as HSI analysis is concerned.

\section{Decision Fusion in Kernel-Induced Space}

\section{A. OAO Strategy}

The OAO strategy [24], which has been applied extensively, divides a multiclass task into binary tasks between all possible pairs of classes. A classifier is trained to discriminate between each pair, and the outputs of these classifiers are then combined to predict the final class label. For this $C$-class problem, $C(C-$ 1) $/ 2$ classifiers represent all possible pairwise classifications. Each classifier considers two information classes $l$ and $s(l, s \in$ $\{1,2, \ldots, C\}, l \neq s)$ via a decision function $g_{l, s}(\mathbf{x})$. The global decision function is then

$$
N_{l}(\mathbf{x})=\sum_{\substack{s=1 \\ s \neq l}}^{C} \operatorname{sgn}\left(g_{l, s}(\mathbf{x})\right)
$$

where $N_{l}(\mathbf{x})$ is the number of times that class $l$ is assigned for the testing vector $\mathrm{x}$. The final class-label decision in the OAO strategy typically follows the MV rule such that the testing sample $\mathbf{x}$ is assigned a final class label according to

$$
y^{*}=\arg \max _{l=1, \ldots, C} N_{l}(\mathbf{x}) .
$$

\section{B. Proposed Classification Framework}

In this paper, we employ the OAO strategy for discriminant analysis in a kernel-induced space. One advantage for doing so is to reduce the high computational cost resulting from the estimation of the kernel matrix during the training process. Specifically, in (7), the size of $K$ is $n \times n$ for samples $\mathbf{X}$, yet the decomposition of a large matrix, if $n$ is sufficiently large, is computationally complex. Suppose that the number of available training samples for the $l$ th class is $n_{l}, \sum_{l=1}^{C} n_{l}=n$. The size of the kernel matrix $K$ in the OAO scheme will instead be $\left(n_{l}+n_{s}\right) \times\left(n_{l}+n_{s}\right)$ which is much smaller than $n \times n$. Hence, the proposed strategy potentially reduces the computational load of the training process.

Furthermore, working with two information classes at a time simplifies the discriminant function, and it is expected that class-conditional statistics will be more separable than in a multiclass setup. The downside is that, in the OAO scheme, the binary classifiers are occasionally forced to assign incorrect class labels. That is, the learning process of a binary classifier may use only the training samples from class $l$ and class $s$; therefore, the decision function $g_{l, s}(\mathbf{x})$ of this binary classifier must force test sample $\mathbf{x}$ into either class $l$ or $s$, even if the sample truly belongs to some other class. However, the MVbased decision fusion in OAO implementations resolves this issue by assigning the class label that receives the highest number of votes [11]. Decision fusion for hyperspectral data analysis can also be found in [25]-[27].

Fig. 1 illustrates the proposed classification framework. The multiclass classifier is partitioned into pairwise two-class problems. For every pair of information classes, the training data are employed to learn a KDA or KLFDA transformation. The transformation then maps the training and testing data into the kernel-induced space before projecting into a 


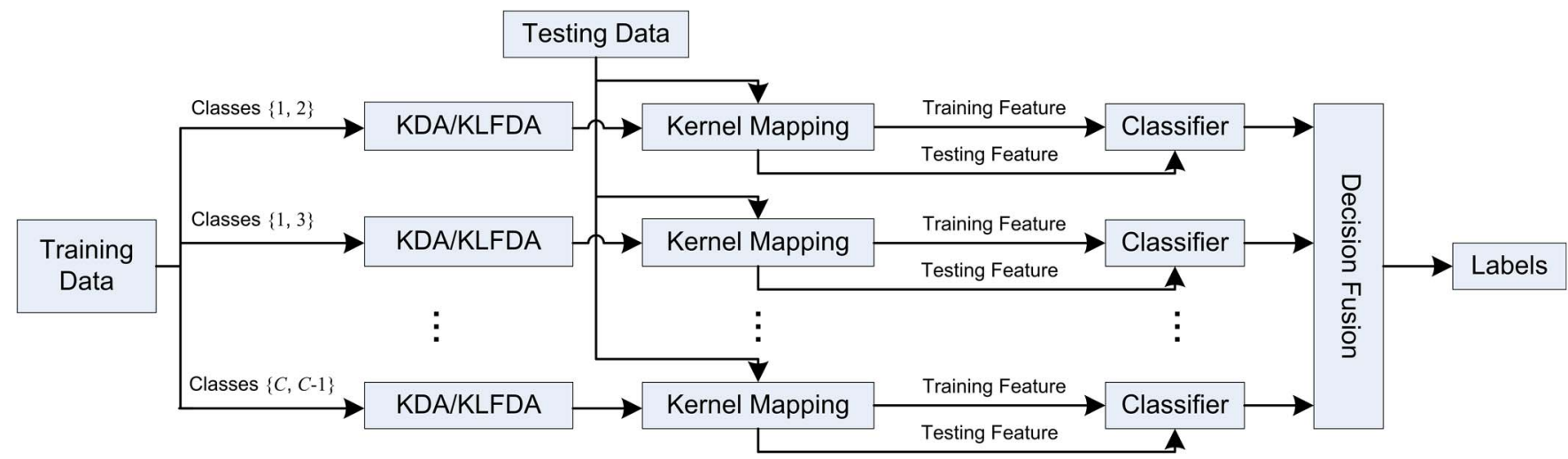

Fig. 1. Proposed pairwise KDA for HSI classification. For a $C$-class problem with $n$ samples, the proposed fusion framework involves $C(C-1) / 2$ classifiers, and the size of the label matrix provided to the decision-fusion process for each testing sample is $(C-1) \times C$.

reduced-dimensional subspace that maximizes a variant of Fisher's ratio under the projection. Finally, a decision-fusion mechanism is employed to merge the classification results from each classifier. Note that, for a $C$-class task, the dimensionality of data in the KDA-projected subspace is $C-1$. Hence, for the binary classification in the OAO scheme (see Fig. 1), the dimensionality of data in the KDA-projected subspace is 1 . However, there is no such restriction for KLFDA since the local between-class scatter matrix generally has a much higher rank than $C-1$ [22].

\section{Classifier and Decision Fusion}

In this paper, we employ the quadratic Gaussian MLE classifier as the base classifier in our framework in Fig. 1. Although other classifiers-such as neural networks, $k$-nearest neighbor, or SVMs - can be employed, it has been observed that the data distribution in kernel-projected spaces tends to be Gaussian [20]. Furthermore, this observation was confirmed by using the Jarque-Bera test [28] which is a goodness-offit measure of departure from a normal distribution. Results from this simple test (MATLAB function JBTEST) reaffirm the choice of the MLE classifier in this paper. In [6], experimental results with hyperspectral data reveal that kernelbased dimensionality-reduction methods followed by an MLE classifier may sometimes outperform SVMs.

The conventional OAO strategy in SVMs makes the final classification decision via a "hard" decision-an MV rulesince only class-label information is available. Such a binary $0-1-\mathrm{MV}$ rule may be suboptimal. In contrast, in this paper, we extend the OAO scheme to employ alternative "soft" decisionfusion approaches, i.e., continuous values. This is possible in proposed framework since the MLE classifier provides posterior-probability estimation which facilitates the use of "soft" decision-fusion schemes such as linear opinion pools (LOPs) and logarithmic opinion pools (LOGPs) [25], [26]. LOP uses the individual posterior probabilities $p_{t}(y \mid \mathbf{x})$ of each classifier to estimate a global membership function-a weighted sum of these output probabilities. The final class label is given according to

$$
y^{*}=\arg \max _{l=1, \ldots, C} P(y \mid \mathbf{x})
$$

where the global membership function is

$$
P(y \mid \mathbf{x})=\sum_{t=1}^{M} \alpha_{t} p_{t}(y \mid \mathbf{x})
$$

and, in this paper, the classifier weights $\left\{\alpha_{t}\right\}_{i=t}^{M}$ are uniformly distributed over all classifiers as in [26], while the number of individual posterior probabilities $\left(p_{t}(y \mid \mathbf{x})\right) M=C-1$ in our case. ${ }^{1}$ Unlike the "hard" decision rule, LOP is appealing in that the output is a combined probability distribution, and the weights $\left\{\alpha_{t}\right\}_{t=1}^{M}$ provide a relative measure for the distribution of the corresponding model. If the posterior probabilities provide continuous probability distributions, the output of LOP produces a continuous distribution as well.

LOP has one potential disadvantage [25]—one posterior probability may dominate the final decision since LOP is simply a weighted average of the different probability distributions. An alternative to the LOP rule is LOGP, which can overcome this problem. In this case, the final class label for LOGP is given according to (11); however, the global membership function for LOGP is

$$
P(y \mid \mathbf{x})=\prod_{t=1}^{M} p_{t}(y \mid \mathbf{x})^{\alpha_{t}}
$$

or

$$
\log P(y \mid \mathbf{x})=\sum_{t=1}^{M} \alpha_{t} p_{t}(y \mid \mathbf{x}) .
$$

As opposed to LOP, the output of LOGP is a weighted product of different probability distributions. A detailed discussion of LOP and LOGP with particular focus on HSI applications can be found in [25] and [26].

\footnotetext{
${ }^{1}$ Since we have $C(C-1) / 2$ binary classifiers and each individual classifier has two output probabilities, for each testing sample, the size of the output matrix is $(C-1) \times C$. In our strategy, the elements in each column of the output matrix are combined using (12), and the number of elements in each column is $M$.
} 


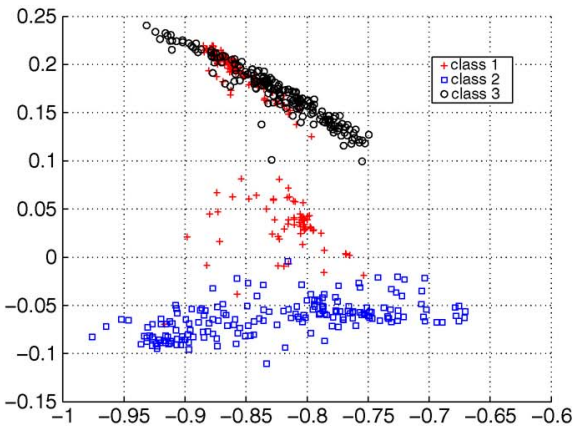

(a)

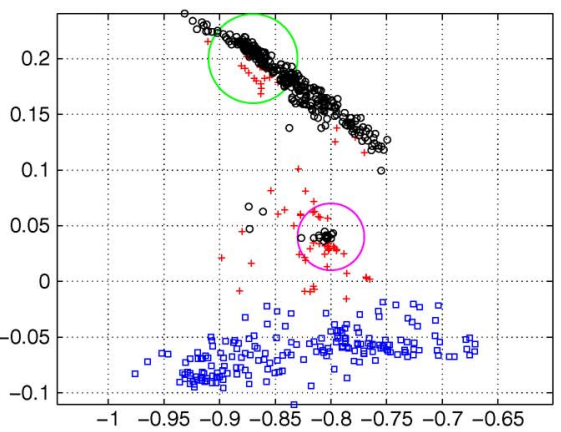

(b)

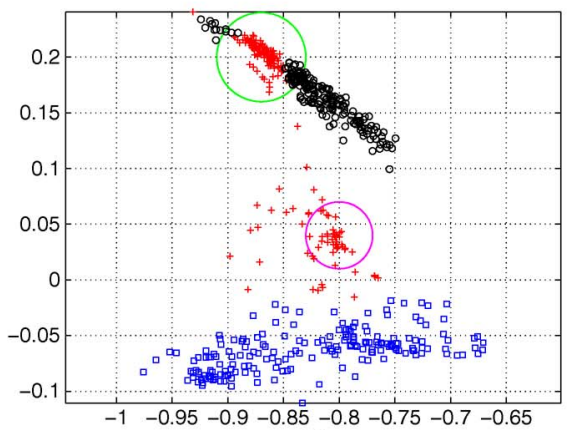

(c)

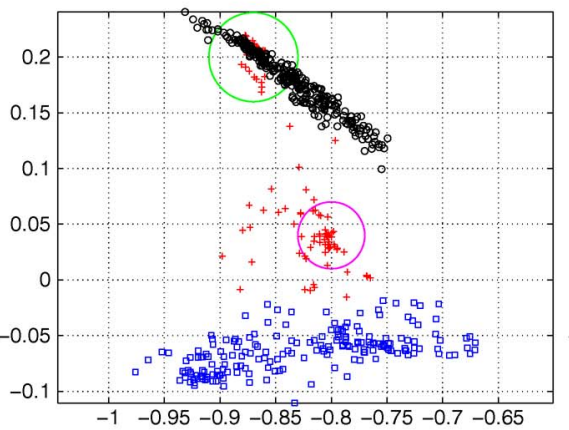

(d)

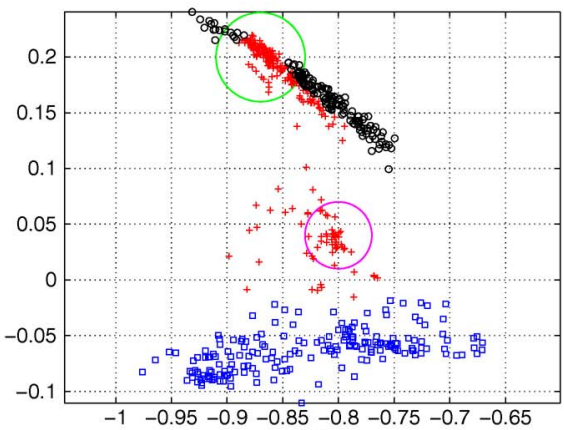

(e)

Fig. 2. Two-dimensional three-class synthetic data classified (with classification accuracy) by traditional KDA-MLE and the proposed KDA-OAO strategy. (a) Original data. (b) KDA-MLE with 73.97\%. (c) KDA-OAO-MV with 84.09\%. (d) KDA-OAO-LOP with 79.14\%. (e) KDA-OAO-LOGP with 87.52\%.

For the remainder of this paper, we use the following notation: the proposed KDA-based OAO schemes are denoted as KDA-OAO-MV, KDA-OAO-LOP, and KDA-OAO-LOGP for the MV, LOP, and LOGP decision-fusion paradigms, respectively. KLFDA-based KLFDA-OAO-MV, KLFDA-OAO-LOP, and KLFDA-OAO-LOGP are defined similarly.

\section{EXPERIMENTAL RESULTS}

To demonstrate the benefits of the proposed classification approach, we present experimental results with synthetic data as well as real HSI data sets representing various ground-cover classification scenarios. The proposed KDA-OAO and KLFDAOAO strategies were compared with conventional algorithms, including LDA, KDA, and KLFDA followed by MLE, RVMs, and SVMs (specifically, SVMs employing the OAO and OAA strategies). Three different decision-fusion rules (MV, LOP, and LOGP) have been tested within the proposed framework. We also compared the OAO strategy with OAA for multiclass discriminant analysis.

\section{A. Synthetic Data Classification}

Two-dimensional three-class synthetic data are simulated as shown in Fig. 2(a). In our example, around 200 samples (data range $[-0.1,0.25])$ are chosen for each class-class 1: red plus, class 2: blue square, and class 3: black circle. The distribution for the synthetic data is complex-class 2 is relatively separable from two others; class 1 mainly has two parts, one of which is significantly overlapped with class 3 . We simply compare the classification performance of the proposed KDA-OAO strategy
TABLE I

ClASS ACCURACIES (\%) FOR CLASSIFIERS USING SYNTHETIC DATA

\begin{tabular}{|c|c|c|c|c|}
\hline & & class 1 & class 2 & class 3 \\
\hline \multirow{2}{*}{$K D A-M L E$} & TPR & 28.88 & 99.47 & 93.58 \\
\hline & FPR & 80.60 & 98.41 & 57.38 \\
\hline \multirow{2}{*}{ KDA-OAO-MV } & TPR & 78.61 & 99.47 & 80.21 \\
\hline & FPR & 79.46 & 98.41 & 80.21 \\
\hline \multirow{2}{*}{$K D A-O A O-L O P$} & TPR & 44.39 & 99.47 & 93.58 \\
\hline & FPR & 86.46 & 98.41 & 63.41 \\
\hline \multirow{2}{*}{ KDA-OAO-LOGP } & TPR & 93.58 & 99.47 & 69.52 \\
\hline & FPR & 75.11 & 98.41 & 93.53 \\
\hline
\end{tabular}

with the traditional KDA. ${ }^{2}$ Leave-one-out cross-validation [29] is considered for the synthetic data. For KDA-MLE, the RBF kernel is used, and the optimal parameter $\sigma$ is chosen to be 0.1 ; for the KDA-OAO strategy, the parameter $\sigma$ is chosen to be 0.07 .

In Fig. 2(b)-(e), the experimental performance of each classifier is provided in terms of the classification map as well as the overall accuracy with each algorithm. Based on the classification maps, we plot two circles-magenta with center $(-0.8$, $0.04)$ and green with center $(-0.87,0.2)$. In this area, we can easily observe the misclassified samples of each classifier, e.g., KDA-MLE wrongly labeled the samples of class 1 as class 3 in the location of the magenta circle. The traditional KDAMLE has $73.97 \%$ accuracy, which is lower than that of the proposed methods. Additionally, we show the true positive ratio (TPR) and false positive ratio (FPR) for each method in Table I. From Table I, the TPR of KDA-MLE for class 1 is clearly low;

\footnotetext{
${ }^{2}$ http://cmp.felk.cvut.cz/cmp/software/stprtool
} 
even though the one for class 3 is high, however, the FPR is relatively low, which causes the overall accuracy to be lower than others. The performance of KDA-OAO-MV is better than KDA-OAO-LOP but worse than KDA-OAO-LOGP. However, comparing the samples under the location of the green circle, these three classifiers have their own advantages. For example, despite a lower classification accuracy, KDA-OAO-LOP is able to partially discriminate some samples from class 1 to class 3.

\section{B. Experimental HSI Data Sets}

Our experimental data were downloaded from the Euskal Herriko Unibertsitatea (EHU) website. ${ }^{3}$ The first HSI data set in this paper was acquired using NASA's Airborne Visible/ Infrared Imaging Spectrometer (AVIRIS) sensor and was collected over northwest Indiana's Indian Pines test site in June 1992. The image represents a vegetation-classification scenario with $145 \times 145$ pixels and 220 spectral bands in the $0.4-2.45-\mu \mathrm{m}$ region of the visible and infrared spectrum with a spatial resolution of $20 \mathrm{~m}$. The main crops of soybean and corn in the image are in their early-growth stage. The notation no till, $\mathrm{min}$ till, and clean till indicate the amount of previous crop residue remaining. There are 16 different land-cover classes in the original ground truth; however, eight classes are used in this study so as to avoid a few classes that have very few training samples [9]. The eight classes used in our experiments are Corn-no till, Corn-min till, Grass/Pasture, Hay-windrowed, Soybean-no till, Soybean-min till, Soybean-clean till, and Woods. There are 187 training samples for each class, and the numbers of testing samples are $1247,647,310,302,781,2281,427$, and 1107 , respectively.

The two other data sets used in this paper were collected by the Reflective Optics System Imaging Spectrometer sensor. The imagery, covering the city of Pavia, Italy, was collected under the HySens project managed by DLR (the German Aerospace Agency). The images have 115 spectral bands with a spectral coverage from 0.43 to $0.86 \mu \mathrm{m}$ and a spatial resolution of $1.3 \mathrm{~m}$. Two scenes are used in our experiment. The first is the university area which has 103 spectral bands with a spatial coverage of $610 \times 340$ pixels. The nine classes used in our experiments are Asphalt, Meadows, Gravel, Trees, Metal Sheets, Bare Soil, Bitumen, Bricks, and Shadows. The numbers of training samples are $185,180,168,176,132,181,163,175$, and 116 , respectively, and the numbers of testing samples are 925 , $900,840,880,660,905,815,875$, and 580 , respectively. The second one is the Pavia city center which has 102 spectral bands with $1096 \times 715$ pixels formed by combining two separate images representing different areas of the Pavia city. The nine classes used in our experiments are Water, Trees, Meadows, Bricks, Bare Soil, Asphalt, Bitumen, Tiles, and Shadows. The numbers of training samples are 165, 164, 165, 162, 164, 163, 162,189 , and 143, respectively, and the numbers of testing samples are 990, 984, 990, 972, 984, 978, 972, 1134, and 858, respectively.

\footnotetext{
${ }^{3}$ http://www.ehu.es/ccwintco/index.php/Hyperspectral_Remote_Sensing Scenes
}

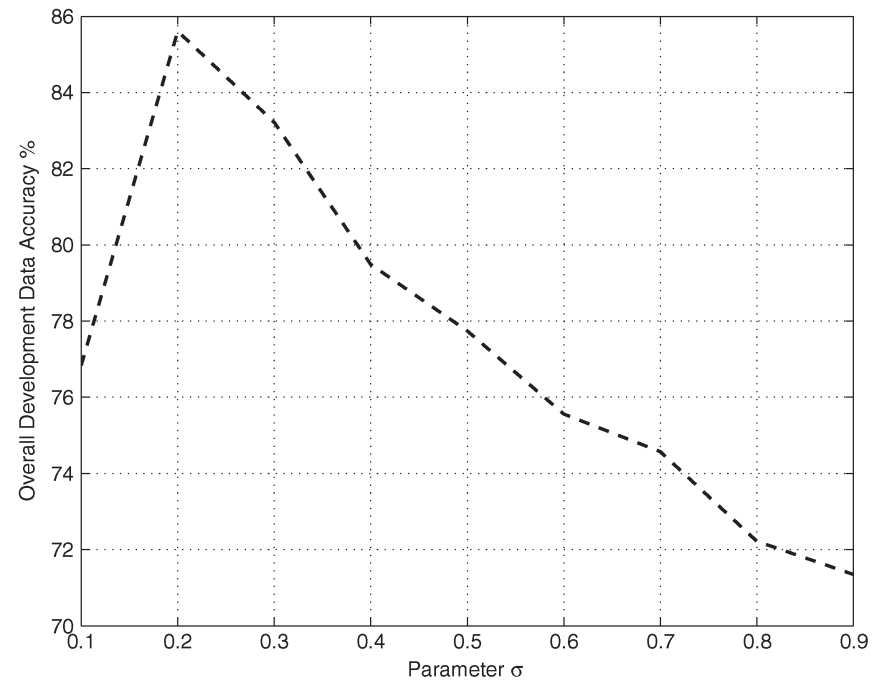

Fig. 3. KDA-OAO-MV using the Indian Pines data set: Overall development accuracy versus parameter $\sigma$.

\section{Parameter Tuning}

We illustrate an optimization strategy for the proposed system. System parameters-such as the reduced dimensionality of the projected subspace and $\sigma$ for the RBF kernel-are optimized using training data. Development data are derived from available training data by further dividing into two groups called "development data for training" and "development data for testing" for tuning these parameters. For example, approximately 500 training samples and 900 testing samples are further randomly chosen from original available training samples for the Indian Pines data set. The testing accuracy obtained using this development data set is used to estimate an effective range for the system parameters to ensure a reliable classification performance.

As we mentioned before, the reduced dimensionality of KDA under the OAO scheme (KDA-OAO-MV) is necessarily 1 . The remaining parameter is then $\sigma$ of the kernel function. For tuning this parameter, a line search over a wide range of possible smoothing parameters is considered. We normalize the data into the range of $[0,1]$, and set the range of smoothing parameters from 0.1 to 1.0 with an interval of 0.1 . Starting from 0.1 , we implement the proposed method using each smoothing parameter in turn, moving at most one grid step up. This is effectively a 1-D grid search for $\sigma$. We compare the classification accuracies among the smoothing parameters in the grid search, finding the optimum point. Fig. 3 illustrates the accuracies of KDA-OAOMV as a function of the parameter $\sigma$. It is clear that the optimal value from the search is $\sigma=0.2$.

For KLFDA under the OAO scheme, Fig. 4 illustrates the overall development-data accuracies of KLFDA-OAO-LOGP as a function of parameter $\sigma$ and the reduced dimension for the Indian Pines data set. We performed a grid search over a wide range of all these system parameters, studying the developmentdata accuracy as a function of these parameters. KLFDA does not have the restriction that the dimensionality is $C-1$ because its between-class scatter matrix has a higher rank than $C-1$ [22]. An interesting observation is that the optimal reduced dimension for KLFDA-OAO-LOGP is still 1, which indicates 


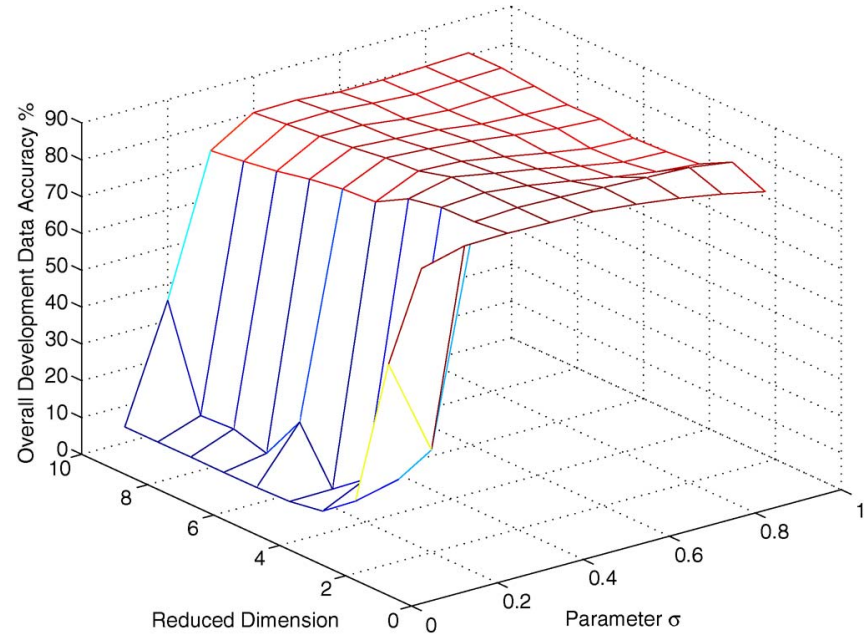

Fig. 4. KLFDA-OAO-LOGP using the Indian Pines data set: Overall development accuracy versus reduced dimension and parameter $\sigma$.

TABLE II

Optimal Parameter $\sigma$ For the Various Algorithms After Tuning

\begin{tabular}{|c|c|c|c|}
\cline { 2 - 4 } \multicolumn{1}{c|}{} & $\begin{array}{c}\text { Indian } \\
\text { Pines }\end{array}$ & $\begin{array}{c}\text { University } \\
\text { of Pavia }\end{array}$ & $\begin{array}{c}\text { Pavia } \\
\text { Centre }\end{array}$ \\
\hline KDA-MLE & 0.1 & 0.6 & 0.3 \\
\hline RVMs & 0.4 & 0.2 & 0.4 \\
\hline SVMs & 0.5 & 0.2 & 0.4 \\
\hline KLFDA-MLE & 0.3 & 0.5 & 0.6 \\
\hline $\begin{array}{c}\text { KDA-OAO } \\
\text { (MV, LOP, LOGP) }\end{array}$ & 0.2 & 0.6 & 0.3 \\
\hline $\begin{array}{c}\text { KLFDA-OAO } \\
\text { (MV, LOP, LOGP) }\end{array}$ & 0.4 & 0.5 & 0.7 \\
\hline
\end{tabular}

TABLE III

RATIOS OF KL DiVERGENCE FOR CLASSES FOR KLFDA-OAO-LOGP AND KLFDA USING INDIAN PINES

\begin{tabular}{|c|c|c|c|c|c|c|c|c|}
\hline Class & 1 & 2 & 3 & 4 & 5 & 6 & 7 & 8 \\
\hline 1 & - & 4.51 & 2.71 & 2.45 & 5.08 & 4.94 & 4.64 & 2.40 \\
\hline 2 & - & - & 1.88 & 2.46 & 4.21 & 4.52 & 5.04 & 2.62 \\
\hline 3 & - & - & - & 2.34 & 3.57 & 2.62 & 2.68 & 2.77 \\
\hline 4 & - & - & - & - & 4.13 & 4.26 & 4.39 & 2.72 \\
\hline 5 & - & - & - & - & - & 4.83 & 5.02 & 2.29 \\
\hline 6 & - & - & - & - & - & - & 4.94 & 2.37 \\
\hline 7 & - & - & - & - & - & - & - & 2.33 \\
\hline 8 & - & - & - & - & - & - & - & - \\
\hline
\end{tabular}

the following: 1) Even in the low-dimensional space, KLFDA exacts the most useful information, including discrimination and locality-preserving properties, and 2) the higher rank of the scatter matrix for KLFDA does not necessarily correspond to better discrimination in a multidimensional subspace. We performed similar tuning experiments for the Pavia data sets and obtained similar results. Table II summarizes the optimal parameters for all proposed methods. More details on the parameter-tuning process for KLFDA can be found in [6].

\section{Benefits of the Proposed Strategy}

Next, we investigate the benefits of the proposed transformation (i.e., the KDA-OAO and KLFDA-OAO strategies). The Kullback-Leibler (KL) divergence [30] is first employed to measure the dissimilarity between distributions for classes after the projection. Table III shows the ratios of KL divergence
TABLE IV

CONFUSION MATRIX FOR KLFDA-MLE USING INDIAN PINES

\begin{tabular}{|c|c|c|c|c|c|c|c|c|}
\hline Class & 1 & 2 & 3 & 4 & 5 & 6 & 7 & 8 \\
\hline 1 & 981 & 83 & 5 & 1 & 71 & 99 & 4 & 0 \\
\hline 2 & 10 & 578 & 0 & 0 & 5 & 47 & 7 & 0 \\
\hline 3 & 0 & 0 & 302 & 0 & 4 & 2 & 2 & 0 \\
\hline 4 & 0 & 0 & 0 & 302 & 0 & 0 & 0 & 0 \\
\hline 5 & 8 & 6 & 5 & 0 & 693 & 60 & 9 & 0 \\
\hline 6 & 106 & 74 & 29 & 0 & 175 & 1870 & 27 & 0 \\
\hline 7 & 0 & 10 & 3 & 2 & 11 & 17 & 384 & 0 \\
\hline 8 & 0 & 0 & 36 & 1 & 0 & 0 & 0 & 1070 \\
\hline \hline TPR & 78.86 & 89.34 & 97.42 & 100 & 88.73 & 81.98 & 89.93 & 96.66 \\
\hline FPR & 88.78 & 76.96 & 79.47 & 98.69 & 72.26 & 89.26 & 88.68 & 100 \\
\hline
\end{tabular}

TABLE V

CONFUSION MATRIX FOR KLFDA-OAO-LOGP USING INDIAN PINES

\begin{tabular}{|c|c|c|c|c|c|c|c|c|}
\hline Class & 1 & 2 & 3 & 4 & 5 & 6 & 7 & 8 \\
\hline 1 & 1054 & 41 & 8 & 0 & 39 & 98 & 7 & 0 \\
\hline 2 & 24 & 564 & 2 & 0 & 2 & 42 & 13 & 0 \\
\hline 3 & 0 & 0 & 301 & 0 & 0 & 1 & 8 & 0 \\
\hline 4 & 0 & 0 & 0 & 302 & 0 & 0 & 0 & 0 \\
\hline 5 & 23 & 8 & 8 & 0 & 666 & 66 & 10 & 0 \\
\hline 6 & 120 & 57 & 33 & 0 & 99 & 1962 & 10 & 0 \\
\hline 7 & 3 & 7 & 5 & 0 & 1 & 13 & 398 & 0 \\
\hline 8 & 0 & 0 & 29 & 1 & 0 & 0 & 0 & 1077 \\
\hline \hline TPR & 84.52 & 87.17 & 97.10 & 100 & 85.02 & 86.76 & 93.21 & 97.29 \\
\hline FPR & 86.11 & 83.31 & 77.98 & 99.67 & 82.53 & 89.92 & 89.24 & 100 \\
\hline
\end{tabular}

for any two classes using KLFDA-OAO-LOGP and KLFDA. All the values, which are larger than one, confirm that the discriminant pattern between classes increases for the proposed transformation as compared to KLFDA. We also show the confusion matrices in Tables IV and V, which reaffirm that the decision-fusion procedure in the OAO strategy is able to overrule incorrect labels introduced by binary classifiers and provide reasonably accurate final class labels. The performances of KLFDA-MLE and the proposed KLFDA-OAO-LOGP are also compared here.

As we have mentioned in Section III-B, the proposed strategy, which breaks down a multiclass problem into all possible binary classifiers, has the benefit of reduced computational cost resulting from the estimation of the kernel matrix during the training process. Specifically, when the total number of training samples $n$ is sufficiently large, the decomposition of a large kernel matrix is essential, which potentially increases computational complexity. As described in Section III-B, under the proposed strategy, the size of the kernel matrix $K$ will instead be $\left(n_{l}+n_{s}\right) \times\left(n_{l}+n_{s}\right)$, which is much smaller than $n \times n$. In this experiment, we plot the computational cost (specifically, the execution time in seconds) for KDA-MLE and KDA-OAO-MV. Both are carried out using MATLAB on an Intel Core2 Duo CPU machine with 4 GB of RAM. As shown in Fig. 5, the execution times for the Indian Pines data set are shown as a function of the number of training samples. In order to demonstrate the benefit of the training process, only a single testing sample for each class (eight classes for these data) is considered. From the results, it can be seen that, even though 28 binary classifiers are employed (note that $C(C-$ 1) $/ 2=8 \times 7 / 2=28$ ), the proposed method requires less time than the traditional KDA-MLE. Furthermore, when the number of training samples increases, the computational benefit 


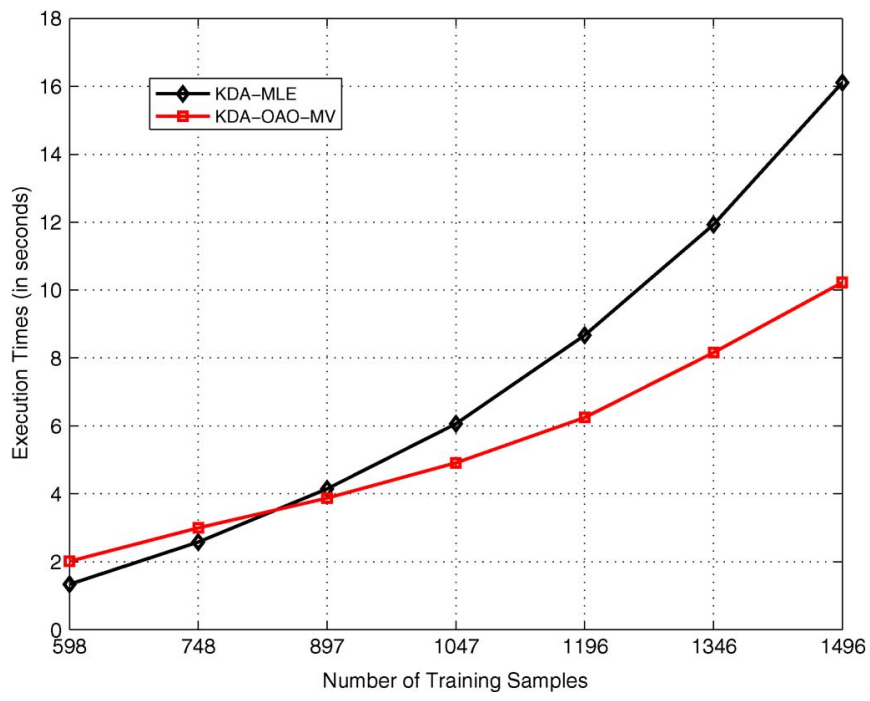

Fig. 5. Execution times versus number of training samples using the Indian Pines data set.

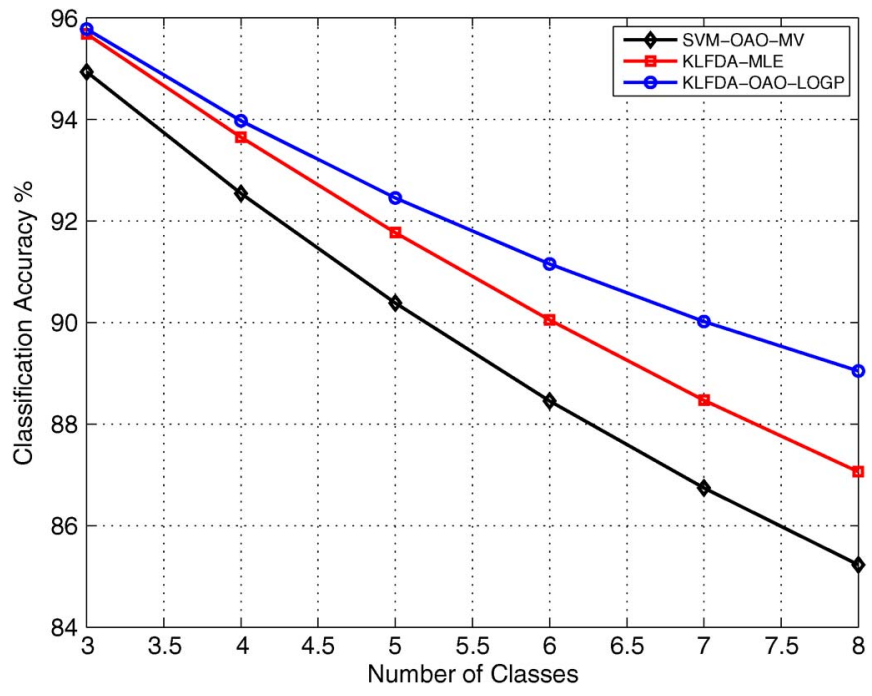

Fig. 6. Overall accuracy versus number of classes using the Indian Pines data set.

of the proposed strategy for the training process is clearly visible.

We also note that, in this paper, we have considered classification tasks involving eight or nine classes. However, this pairwise kernel-discriminant approach can indeed be scaled up to complicated scenes involving many more classes. We demonstrate that the gain in classification performance, in fact, increases with the number of classes. Consider the Indian Pines data set with eight classes for example; Fig. 6 shows the overall classification as the number of classes ranges from three to eight. We list all the possible cases (e.g., $\left(\begin{array}{l}8 \\ 4\end{array}\right)=70$ for four classes) for choosing the number of classes and average the classification accuracies. Three techniques (SVM-OAO-MV, KLFDA-MLE, and KLFDA-OAO-LOGP) are considered here. From the results, when the number of classes is three, the accuracies of KLFDA-MLE and KLFDA-OAO-LOGP are very close, which both are slightly better than that of SVM-OAO-

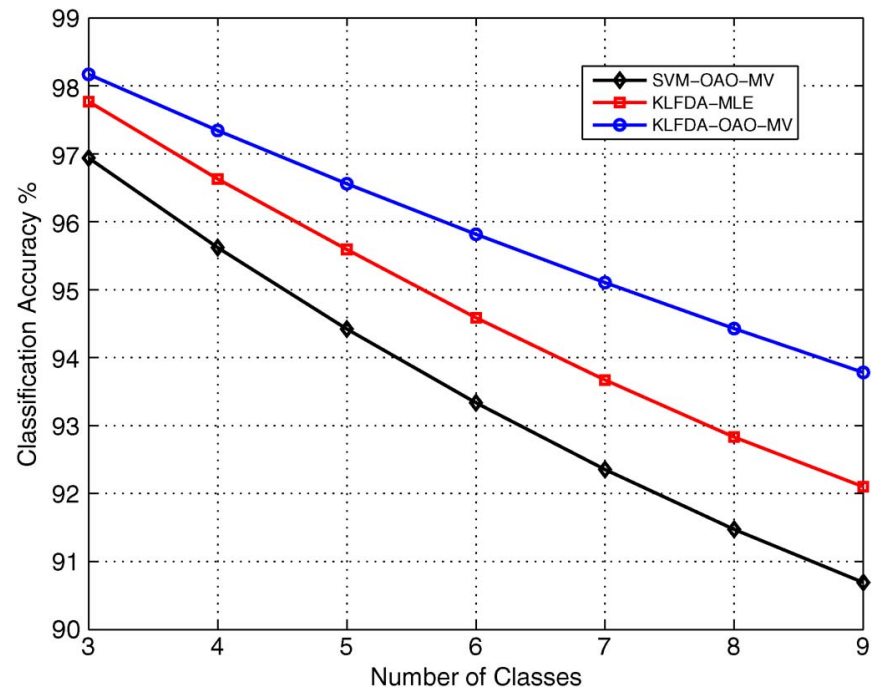

Fig. 7. Overall accuracy versus number of classes using the Pavia Centre data set.

MV. Nevertheless, when the number of classes increases, the performance gap between the proposed KLFDA-OAO-LOGP and traditional KLFDA-MLE is more pronounced. Fig. 7 illustrates the overall classification as the number of classes ranges from three to nine for the Pavia Centre data set, which indicates that similar results have been observed for other experimental hyperspectral data sets.

\section{E. HSI Data Classification}

We now evaluate the classification performance of the proposed strategies using real hyperspectral data. The classification accuracies of the proposed approaches as well as those of the current state-of-the-art techniques for each class are shown in Table VI for the Indian Pines data set. For this data set, KLFDAOAO-LOGP provides the best overall classification accuracy compared to the other methods such as KLFDA and SVMs. For class 6 (Soybean-min till), the accuracy is approximately $11 \%$ higher than that of SVMs. There is no significant improvement for KDA-OAA-MV (KDA with a MLE classifier and OAA decision fusion) and KLFDA-OAA-MV. Similar results were obtained for the Pavia data sets as can be seen in Tables VII and VIII. From these results, it is clear that the proposed methods outperform traditional LDA, KDA, and KLFDA followed by MLE, as well as SVMs. For this data set, KLFDA-OAO-MV yields the best overall performance. Interestingly, the performance of KLFDA-OAA-MV is even worse than that of the previously introduced KLFDA-MLE [6]. Take class 5 (Metal Sheets) of the University of Pavia data set as an example-the classification accuracy is $17 \%$ lower than that of KLFDA-MLE.

We also report ground-cover classification maps for the experimental data sets-Fig. 8 illustrates the thematic maps resulting from the classification of these hyperspectral scenes using LDA-MLE, SVMs, KLFDA-MLE, and KLFDA-OAOLOGP. We produced ground-cover maps of the entire HSI scene for these images (including unlabeled pixels). However, 
TABLE VI

ClassificAtion ACCURACIES (\%) USING INDiAn Pines

\begin{tabular}{|c||c|c|c|c|c|c|c|c||c|}
\hline Class & 1 & 2 & 3 & 4 & 5 & 6 & 7 & 8 & Overall \\
\hline LDA-MLE & 71.69 & 71.87 & 97.06 & 100 & 77.59 & 60.41 & 91.10 & 98.92 & 76.51 \\
KDA-MLE & 74.01 & 82.07 & 95.16 & 100 & 82.07 & 81.94 & 83.61 & 89.52 & 83.20 \\
RVM-OAO-MV & 81.64 & 85.94 & 95.16 & 100 & 81.95 & 72.60 & 89.93 & 98.28 & 84.37 \\
SVM-OAO-MV & 81.47 & 86.55 & 97.09 & 100 & 87.32 & 74.44 & 93.21 & 99.01 & 85.22 \\
SVM-OAA-MV & 80.99 & 84.39 & 98.06 & 100 & 89.24 & 72.49 & 96.02 & 99.19 & 84.81 \\
KLFDA-MLE & 78.91 & $\mathbf{8 9 . 3 4}$ & 97.42 & 100 & 88.73 & 81.98 & 89.93 & 96.66 & 87.06 \\
KDA-OAA-MV & 81.80 & 78.05 & 85.16 & 93.38 & 81.31 & $\mathbf{8 6 . 7 6}$ & 77.75 & 82.29 & 83.47 \\
KLFDA-OAA-MV & 83.40 & 84.08 & 95.81 & 100 & 89.24 & 80.97 & 88.99 & 98.64 & 87.29 \\
\hline KDA-OAO-MV & 84.12 & 88.72 & 97.09 & 100 & 83.09 & 75.40 & 91.57 & 98.82 & 85.61 \\
KDA-OAO-LOP & 81.64 & 83.77 & 96.45 & 100 & 81.82 & 78.39 & 89.46 & 96.75 & 85.06 \\
KDA-OAO-LOGP & 80.35 & 83.46 & 96.45 & 100 & 81.82 & 80.75 & 88.99 & 96.66 & 85.53 \\
KLFDA-OAO-MV & $\mathbf{8 5 . 1 6}$ & 88.71 & 97.42 & 100 & $\mathbf{9 0 . 1 4}$ & 78.56 & 92.97 & 98.64 & 87.65 \\
KLFDA-OAO-LOP & 84.84 & 87.48 & $\mathbf{9 7 . 7 4}$ & 100 & 85.92 & 85.01 & $\mathbf{9 3 . 2 1}$ & 97.11 & 88.87 \\
KLFDA-OAO-LOGP & 84.52 & 87.17 & 97.10 & 100 & 85.02 & $\mathbf{8 6 . 7 6}$ & 93.21 & 97.29 & $\mathbf{8 9 . 0 5}$ \\
\hline
\end{tabular}

TABLE VII

Classification ACCURACIES (\%) Using University OF PAVIA

\begin{tabular}{|c||c|c|c|c|c|c|c|c|c||c|}
\hline Class & 1 & 2 & 3 & 4 & 5 & 6 & 7 & 8 & 9 & Overall \\
\hline LDA-MLE & 82.27 & 80.44 & 74.04 & 96.47 & 99.03 & 89.83 & 82.33 & 70.63 & 98.62 & 85.23 \\
KDA-MLE & 74.92 & 74.44 & 75.00 & 93.75 & 99.09 & 85.96 & 93.74 & 80.34 & 99.83 & 86.31 \\
RVM-OAO-MV & 85.84 & 88.44 & 82.50 & 91.93 & 99.24 & 89.39 & 93.87 & 86.74 & 99.48 \\
SVM-OAO-MV & 85.62 & 82.55 & 82.02 & 94.20 & 93.48 & 87.62 & 92.14 & 84.34 & 98.96 \\
SVM-OAA-MV & 84.54 & 83.22 & 83.21 & 95.57 & 96.97 & 85.97 & 83.01 & 85.26 & 99.48 & 89.43 \\
KLFDA-MLE & 88.86 & 90.89 & 73.33 & 93.64 & 98.48 & 91.93 & 88.71 & 81.25 & 99.82 & 90.21 \\
KDA-OAA-MV & 84.02 & 91.11 & $\mathbf{8 5 . 0 1}$ & 89.20 & 88.94 & 88.95 & 91.53 & 84.57 & 96.72 & 88.55 \\
KLFDA-OAA-MV & 89.95 & 94.89 & 79.05 & 92.50 & 81.67 & 90.72 & 84.17 & 86.46 & 99.31 & 88.89 \\
\hline KDA-OAO-MV & 84.01 & 85.55 & 82.74 & 96.36 & 99.24 & 88.17 & $\mathbf{9 4 . 6 0}$ & 87.54 & 100 & 90.24 \\
KDA-OAO-LOP & 90.81 & 79.22 & 80.12 & 95.91 & 98.79 & 90.94 & 86.99 & 84.11 & 99.83 & 89.01 \\
KDA-OAO-LOGP & 94.16 & 84.44 & 74.76 & 91.14 & 98.79 & 90.72 & 84.72 & 87.54 & 99.83 & 88.91 \\
KLFDA-OAO-MV & 86.81 & $\mathbf{9 2 . 7 8}$ & 82.97 & 95.57 & 99.94 & 92.92 & 94.23 & 86.29 & 100 & $\mathbf{9 2 . 6 4}$ \\
KLFDA-OAO-LOP & 92.75 & 88.44 & 83.57 & 95.23 & 98.64 & 92.48 & 93.78 & 83.88 & 99.83 & 91.86 \\
KLFDA-OAO-LOGP & 92.43 & 88.89 & 81.66 & 95.00 & 96.85 & 92.26 & 94.04 & 84.34 & 99.84 & 91.48 \\
\hline
\end{tabular}

TABLE VIII

Classification Accuracies (\%) Using Pavia Centre

\begin{tabular}{|c||c|c|c|c|c|c|c|c|c||c|}
\hline Class & 1 & 2 & 3 & 4 & 5 & 6 & 7 & 8 & 9 & Overall \\
\hline LDA-MLE & 99.79 & 80.08 & 97.07 & 84.05 & 76.11 & 88.13 & 77.77 & 98.85 & 97.90 & 88.94 \\
KDA-MLE & 100 & 76.82 & 93.53 & 77.88 & 87.91 & 93.76 & 82.82 & 98.67 & 97.20 & 89.92 \\
RVM-OAO-MV & 100 & 78.86 & 97.37 & 76.44 & 87.09 & 92.94 & 75.51 & 98.68 & 100 & 89.71 \\
SVM-OAO-MV & 100 & 78.25 & 97.17 & 77.26 & 83.74 & 94.27 & 86.21 & 98.94 & 100 & 90.69 \\
SVM-OAA-MV & 100 & 77.85 & 97.27 & 78.60 & 85.57 & 94.48 & 84.57 & 98.41 & 100 & 90.78 \\
KLFDA-MLE & 100 & 81.60 & 97.97 & 82.09 & 90.75 & 94.37 & 82.82 & 99.12 & 100 & 92.10 \\
KDA-OAA-MV & 99.70 & 73.88 & 97.68 & 76.85 & 82.62 & 92.23 & 85.19 & 97.44 & 92.77 & 88.83 \\
KLFDA-OAA-MV & 100 & 84.04 & 95.56 & 80.45 & 86.28 & 92.33 & 80.86 & 98.32 & 99.42 & 90.85 \\
\hline KDA-OAO-MV & 100 & 79.67 & 96.76 & 78.40 & 92.88 & 93.35 & 82.82 & 99.12 & 100 & 91.49 \\
KDA-OAO-LOP & 100 & 79.57 & 95.76 & 75.62 & 90.96 & 92.13 & 86.32 & 98.85 & 100 & 91.06 \\
KDA-OAO-LOGP & 100 & 79.67 & 95.76 & 75.82 & 90.75 & 92.64 & 85.80 & 98.85 & 100 & 91.07 \\
KLFDA-OAO-MV & 100 & $\mathbf{8 6 . 0 7}$ & 98.68 & $\mathbf{8 9 . 4 0}$ & $\mathbf{9 4 . 4 1}$ & 92.74 & 86.71 & 98.94 & 100 \\
KLFDA-OAO-LOP & 100 & 84.77 & 97.55 & 86.25 & 93.19 & 92.12 & $\mathbf{8 7 . 1 3}$ & 98.94 & 100 \\
KLFDA-OAO-LOGP & 100 & 84.16 & 97.05 & 86.26 & 92.58 & $\mathbf{9 4 . 0 1}$ & 86.01 & 98.41 & 100 & 94.29 \\
\hline
\end{tabular}

to facilitate comparison between methods, only areas for which we have ground truth are shown. Clearly, KLFDA-OAO-LOGP results in a classification map that is least noisy and most accurate compared to traditional state-of-the-art methods. Figs. 9 and 10 further illustrate the thematic maps resulting from the classification of these hyperspectral images in areas for which we have ground truth. These maps are consistent with the results shown in Tables VII and VIII.

Based on these classification maps, we further perform McNemar's test [31], which is based on a standardized normal test statistic

$$
Z=\frac{f_{12}-f_{21}}{\sqrt{f_{12}+f_{21}}}
$$

where $f_{12}$ indicates the number of samples classified correctly by classifier 1 and simultaneously misclassified by classifier 2 . The values of $Z$ larger than 1.96 and 2.58 indicate that two classification results are significantly different at the $95 \%$ and 99\% confidence levels, respectively. The sign of $Z$ indicates whether classifier 1 outperforms classifier $2(Z>0)$ or vice versa. Table IX shows the statistical significance of the difference between the proposed KLFDA-OAO-LOGP (classifier 1) and traditional KLFDA-MLE (classifier 2) for the Indian Pines data set (the whole scenario with ground truth). Table $\mathrm{X}$ shows the McNemar's test between the proposed KLFDA-OAO-MV (classifier 1) and KLFDA-MLE (classifier 2) for the University of Pavia data set. The overall results of McNemar's test confirm the conclusions of previous experiments. 


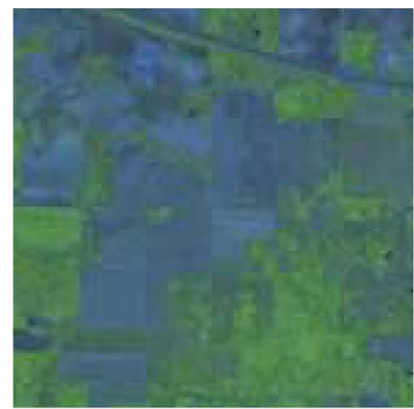

(a)

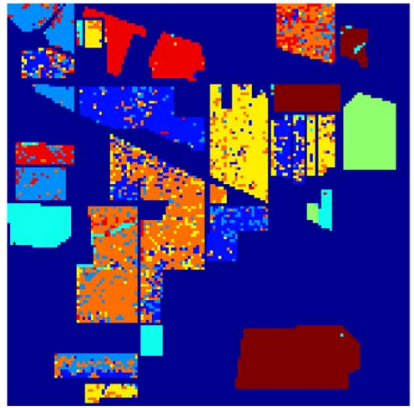

(c)

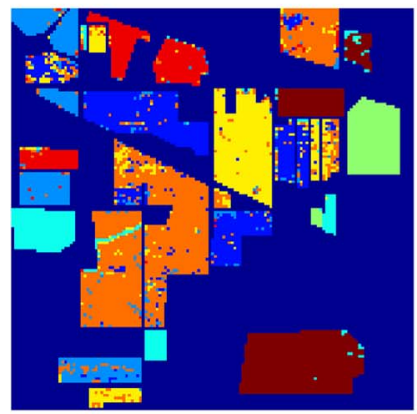

(e)

\begin{tabular}{|c|c|c|c|}
\hline & & & \\
\hline Corn-no till & Corn-min till & Grass/Pasture & Hay-windowed \\
\cline { 2 - 4 } & & & \\
\hline Soybean-no till & Soybean-min till & Soybean-clean till & Woods \\
\hline
\end{tabular}

Fig. 8. Thematic maps resulting from classification using the Indian Pines data set. (a) False-color image. (b) Ground truth. (c) LDA-MLE. (d) SVMOAO-MV. (e) KLFDA-MLE. (f) KLFDA-OAO-LOGP.

In practice, often only a few training samples of hyperspectral data are available. Here, we report the overall accuracy of various classification systems studied in this paper as a function of the number of training samples employed for the Indian Pines data set in Fig. 11. Similar results were obtained with the Pavia data sets. To avoid any spatial biases, we randomly choose a subset of training samples for each samplesize value and repeat the experiment 20 times, reporting the average classification accuracy. It is obvious from Fig. 11 that the accuracy of all algorithms decreases monotonically as the number of training samples decreases. However, the rates at which the accuracy drops for KDA-OAO-MV and KLFDAOAO-LOGP are much slower than that of the other baseline methods when the number of training samples is reduced. This illustrates another benefit of the KDA-OAO and KLFDAOAO approaches-the resulting classifier becomes more robust under the small training-size classification scenario.

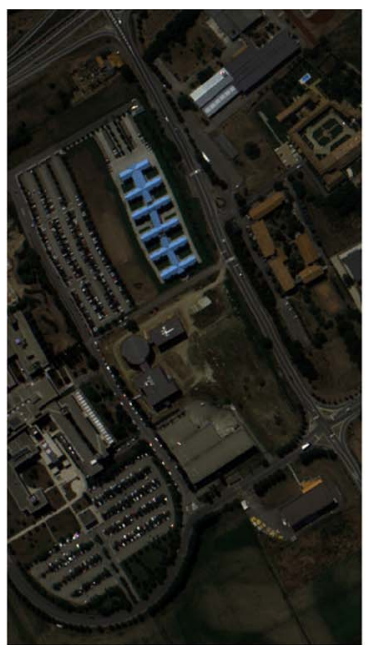

(a)

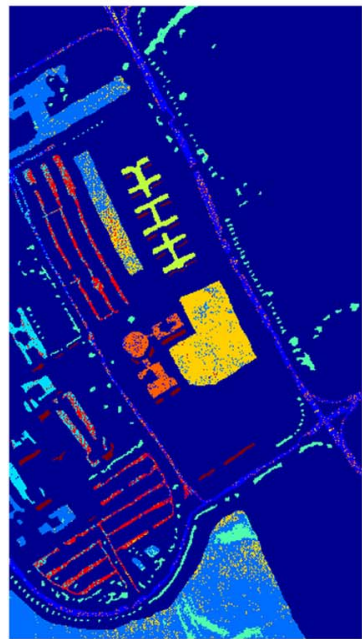

(c)

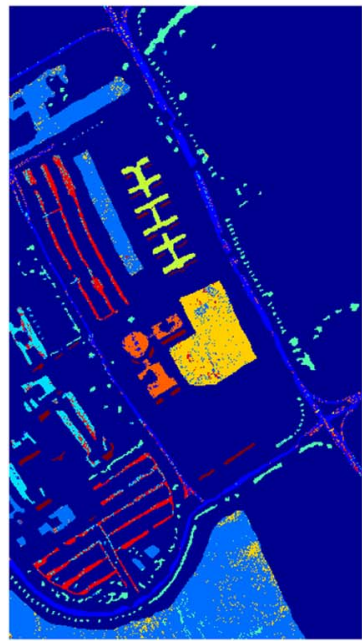

(e)

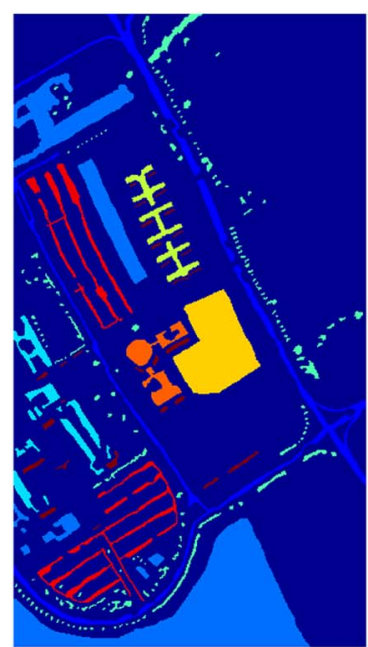

(b)

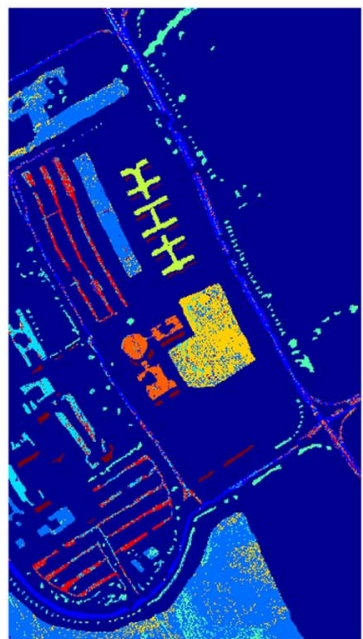

(d)

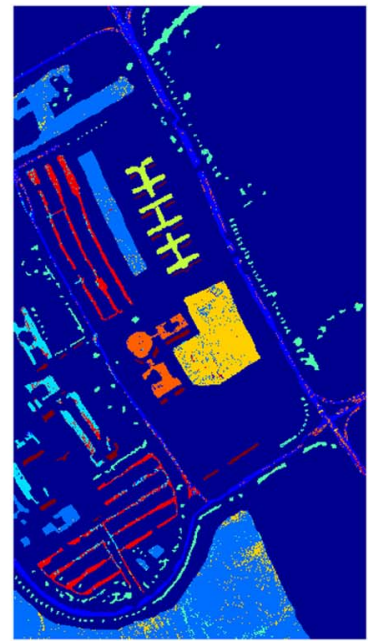

(f)

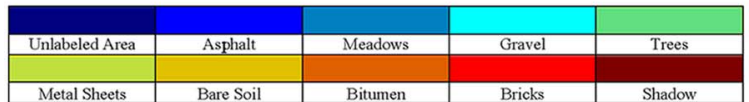

Fig. 9. Thematic maps resulting from classification using the University of Pavia data set. (a) False-color image. (b) Ground truth. (c) LDA-MLE. (d) SVM-OAO-MV. (e) KLFDA-MLE. (f) KLFDA-OAO-MV. 


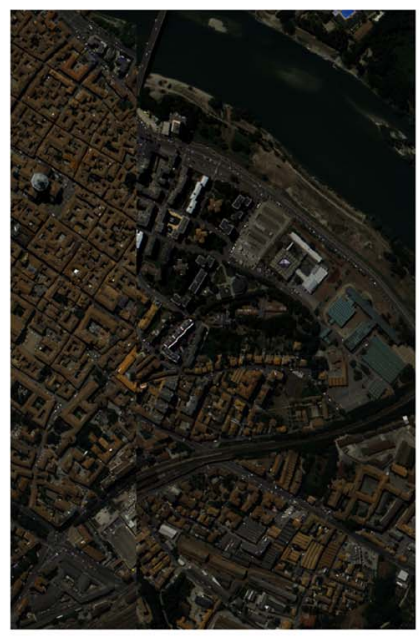

(a)

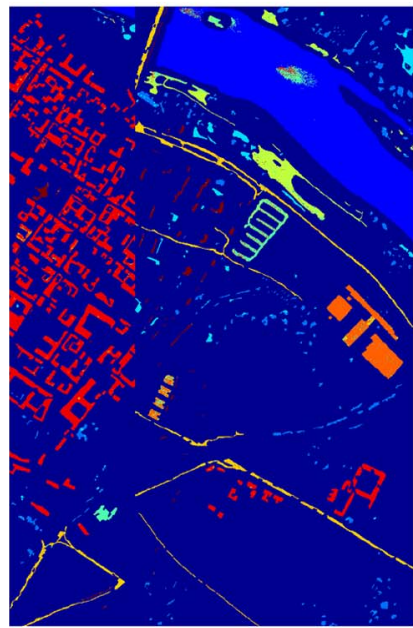

(c)

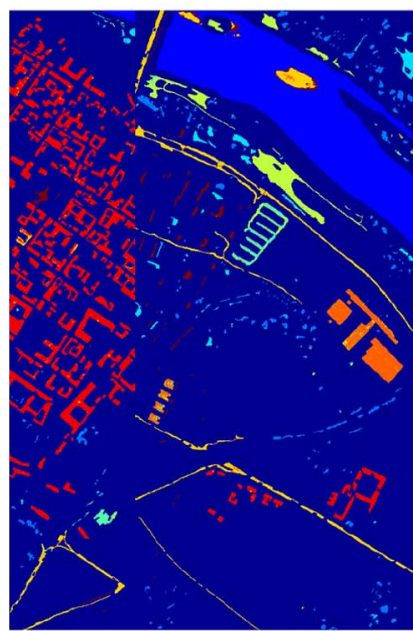

(e)

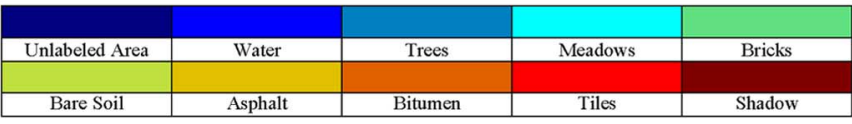

Fig. 10. Thematic maps resulting from classification using the Pavia Centre data set. (a) False-color image. (b) Ground truth. (c) LDA-MLE. (d) SVMOAO-MV. (e) KLFDA-MLE. (f) KLFDA-OAO-MV.
TABLE IX

Statistical Significance of THE DifFERENCE BeTweEn Two ClASSIFIERS-KLFDA-OAO-LOGP (CLASSIFIER 1) AND KLFDA-MLE (Classifier 2)-Using the Indian Pines Data Set

\begin{tabular}{|c|c|c|c|c||c|}
\hline Class & $f_{12}$ & $f_{21}$ & $f_{12}-f_{21}$ & $\sqrt{f_{12}+f_{21}}$ & $Z$ \\
\hline 1 & 115 & 48 & 67 & 12.76 & 5.24 \\
\hline 2 & 25 & 40 & -15 & 8.06 & -1.86 \\
\hline 3 & 1 & 0 & 1 & 1.00 & 1.00 \\
\hline 4 & 0 & 0 & 0 & 0 & NaN \\
\hline 5 & 28 & 58 & -30 & 9.27 & -3.23 \\
\hline 6 & 191 & 102 & 89 & 17.11 & 5.19 \\
\hline 7 & 19 & 7 & 12 & 5.09 & 2.35 \\
\hline 8 & 8 & 3 & 5 & 3.31 & 1.50 \\
\hline \hline Overall & 387 & 258 & 129 & 25.39 & $\mathbf{5 . 0 7}$ \\
\hline
\end{tabular}

TABLE $X$

Statistical Significance of the Difference Between Two CLASSIFIERS-KLFDA-OAO-MV (CLASSIFIER 1) AND KLFDA-MLE

(Classifier 2)-USing the University of Pavia Data Set

\begin{tabular}{|c|c|c|c|c||c|}
\hline Class & $f_{12}$ & $f_{21}$ & $f_{12}-f_{21}$ & $\sqrt{f_{12}+f_{21}}$ & $Z$ \\
\hline 1 & 220 & 324 & -104 & 23.32 & -4.45 \\
\hline 2 & 313 & 307 & 6 & 24.89 & 0.24 \\
\hline 3 & 144 & 91 & 53 & 15.32 & 3.45 \\
\hline 4 & 211 & 5 & 206 & 14.69 & 14.01 \\
\hline 5 & 75 & 0 & 75 & 8.66 & 8.66 \\
\hline 6 & 183 & 121 & 62 & 17.43 & 3.55 \\
\hline 7 & 100 & 9 & 91 & 10.44 & 7.65 \\
\hline 8 & 270 & 119 & 151 & 19.72 & 7.65 \\
\hline 9 & 1 & 0 & 1 & 1.00 & 1.00 \\
\hline \hline Overall & 1517 & 976 & 541 & 49.93 & $\mathbf{1 0 . 8 3}$ \\
\hline
\end{tabular}

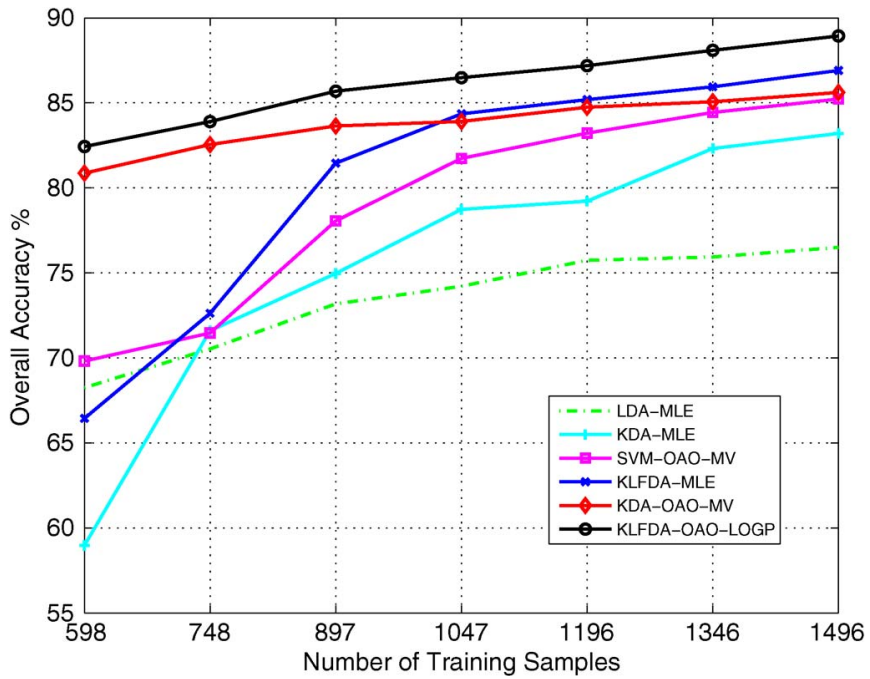

Fig. 11. Effect of number of training samples using the Indian Pines data set.

We also demonstrate another advantage of the KDA-OAO and KLFDA-OAO approaches-reliable performance even under the presence of additive noise [15]. We assume that noise is independent of the signal information [32], [33] and add different amounts of Gaussian noise to both the training and testing samples; the signal-to-noise ratio (SNR) [34] is used to measure the noise level. We varied the SNR between 15 and $35 \mathrm{~dB}$, and 20 realizations were averaged for estimating the average accuracy at each SNR. From Fig. 12, we observe that KLFDA-OAO-LOGP yields the best performance for over a wide range of SNRs. KDA-OAO-MV also shows higher overall accuracy than other previously proposed methods. 


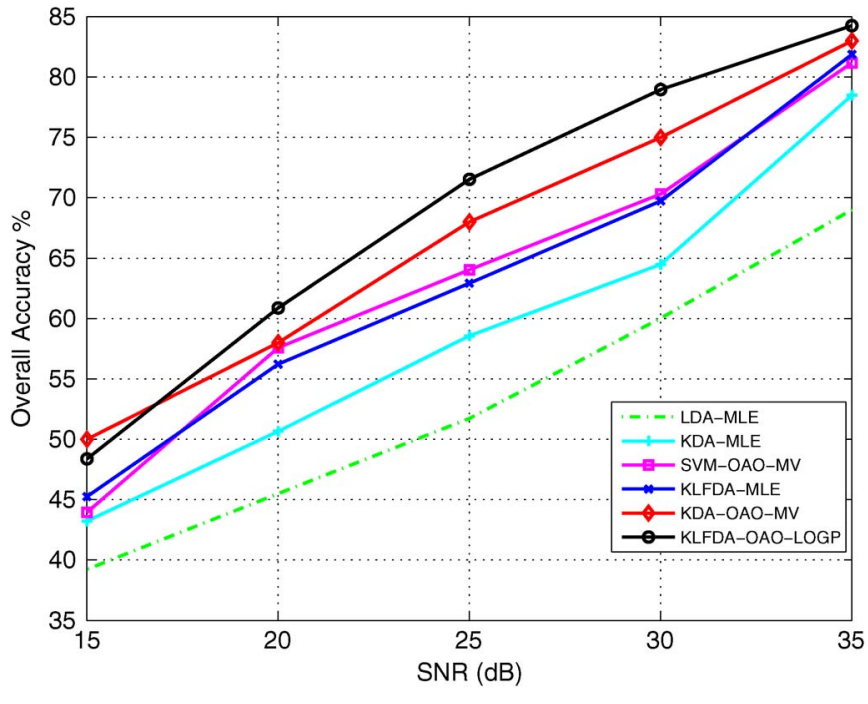

Fig. 12. Behavior of the overall accuracy versus different amounts of noise added using the Indian Pines data set.

\section{CONCLUSiON}

In this paper, we studied a combination of the OAO strategy with kernel-based discriminant analysis for HSI classification. Although OAO has been implemented and commonly used for SVMs, its benefits for KDA and KLFDA have not been studied before. Kernel methods have the advantage of being able to extract nonlinear features from hyperspectral data, but they increase computational load. The proposed OAO strategy alleviated this problem, resulting in robust performance even under very small training-sample sizes, as well as reduced computational requirements when the number of training samples is large. Under the proposed strategy, multiclass discriminant analysis was converted into several two-class problems in the kernel-induced space, and the final multiclass prediction was derived from the predictions of pairwise classifiers. Although the reduced dimensionality of kernel-induced feature subspaces under the OAO scheme may be restricted to 1 , such as was the case for KDA, the decision-fusion procedure still provided highly accurate classification.

Three hyperspectral data sets (one representing vegetation classification and the other two representing urban classification) were used to evaluate the proposed strategy, the performance of which was compared with that of traditional methods. Experimental results demonstrated that the proposed KDA-OAO-MV, KLFDA-OAO-MV, KLFDA-OAO-LOP, and KLFDA-OAO-LOGP techniques always yielded highly accurate classification results as well as outperformed popular algorithms. It is worthwhile mentioning that, for the AVIRIS Indian Pines data set, the "soft" LOGP decision-fusion rule had superior performance compared to the "hard" MV rule. Furthermore, a small number of training samples were needed to obtain accurate classification performance owing to the highly reduced dimensionality. Another interesting observation is that there was no substantial benefit in combining the OAO scheme with some linear classification methods, such as the traditional LDA followed by the MLE classifier-the benefit accrued only when employing KDA or KLFDA as the preprocessing.

\section{REFERENCES}

[1] R. O. Duda, P. E. Hart, and D. G. Stork, Pattern Classification, 2nd ed. New York, NY, USA: Wiley, 2001.

[2] Y. Xu, D. Zhang, Z. Jin, M. Li, and J. Y. Yang, "A fast kernel-based nonlinear discriminant analysis for multi-class problems," Pattern Recognit., vol. 39, no. 6, pp. 1026-1033, Jun. 2006.

[3] S. Mika, G. Rätsch, J. Weston, B. Schölkopf, A. Smola, and K. R. Müller, "Constructing descriptive and discriminative nonlinear features: Rayleigh coefficients in kernel feature spaces," IEEE Trans. Pattern Anal. Mach. Intell., vol. 25, no. 5, pp. 623-628, May 2003.

[4] S. Prasad and L. Mann Bruce, "Information fusion in kernel-induced spaces for robust subpixel hyperspectral ATR," IEEE Geosci. Remote Sens. Lett., vol. 6, no. 3, pp. 572-576, Jul. 2009.

[5] Y. Gu, Y. Liu, and Y. Zhang, "A selective KPCA algorithm based on highorder statistics for anomaly detection in hyperspectral imagery," IEEE Geosci. Remote Sens. Lett., vol. 5, no. 1, pp. 43-47, Jan. 2008.

[6] W. Li, S. Prasad, J. E. Fowler, and L. Mann Bruce, "Locality-preserving discriminant analysis in kernel-induced spaces for hyperspectral image classification," IEEE Geosci. Remote Sens. Lett., vol. 8, no. 5, pp. 894898, Sep. 2011.

[7] T. V. Bandos, L. Bruzzone, and G. Camps-Valls, "Classification of hyperspectral images with regularized linear discriminant analysis," IEEE Trans. Geosci. Remote Sens., vol. 47, no. 3, pp. 862-873, Mar. 2009.

[8] E. Pekalska and B. Haasdonk, "Kernel discriminant analysis for positive definite and indefinite kernels," IEEE Trans. Pattern Anal. Mach. Intell., vol. 31, no. 6, pp. 1017-1032, Jun. 2009.

[9] F. Melgani and L. Bruzzone, "Classification of hyperspectral remote sensing images with support vector machines," IEEE Trans. Geosci. Remote Sens., vol. 42, no. 8, pp. 1778-1790, Aug. 2004.

[10] C. Cortes and V. N. Vapnik, "Support vector networks," Mach. Learn., vol. 20, no. 3, pp. 273-297, Sep. 1995.

[11] N. García-Pedrajas and D. Ortiz-Boyer, "Improving multiclass pattern recognition by the combination of two strategies," IEEE Trans. Pattern Anal. Mach. Intell., vol. 28, no. 6, pp. 1001-1006, Jun. 2006.

[12] C. W. Hsu and C. J. Lin, "A comparison of methods for multiclass support vector machines," IEEE Trans. Neural Netw., vol. 13, no. 2, pp. 415-425, Mar. 2002.

[13] B. Liu, Z. Hao, and E. C. C. Tsang, "Nesting one-against-one algorithm based on SVMs for pattern classification," IEEE Trans. Neural Netw., vol. 19, no. 12, pp. 2044-2052, Dec. 2008.

[14] G. Yang, H. Liu, and X. Yu, "Hyperspectral remote sensing image classification based on kernel Fisher discriminant analysis," in Proc. Int. Conf. Wavelet Anal. Pattern Recognit., Beijing, China, Nov. 2007, pp. 1139-1143.

[15] G. Camps-Valls and L. Bruzzone, "Kernel-based methods for hyperspectral image classification," IEEE Trans. Geosci. Remote Sens., vol. 43, no. 6, pp. 1351-1362, Jun. 2005.

[16] D. Tuia, G. Camps-Valls, G. Matasci, and M. Kanevski, "Learning relevant image features with multiple-kernel classification," IEEE Trans. Geosci. Remote Sens., vol. 48, no. 10, pp. 3780-3791, Oct. 2010.

[17] B. Demir and S. Ertürk, "Hyperspectral image classification using relevance vector machines," IEEE Geosci. Remote Sens. Lett., vol. 4, no. 4, pp. 586-590, Oct. 2007.

[18] F. A. Mainji and Y. Zhang, "Robust hyperspectral classification using relevance vector machine," IEEE Trans. Geosci. Remote Sens., vol. 49, no. 6, pp. 2100-2112, Jun. 2011.

[19] A. Zare and P. Gader, "Hyperspectral band selection and endmember detection using sparsity promoting priors," IEEE Geosci. Remote Sens. Lett., vol. 5, no. 2, pp. 256-260, Apr. 2008.

[20] S. Y. Huang and C. R. Hwang, "Kernel Fisher discriminant analysis in Gaussian reproducing kernel Hilbert spaces,” Inst. Stat. Sci., Acad. Sinica, Taipei, Taiwan, 2006, Tech. Rep.

[21] B. Chen, H. Liu, and Z. Bao, "A kernel optimization method based on the localized kernel Fisher criterion," Pattern Recognit., vol. 41, no. 3, pp. 1098-1109, Mar. 2008.

[22] M. Sugiyama, "Dimensionality reduction of multimodal labeled data by local Fisher discriminant analysis," J. Mach. Learn. Res., vol. 8, no. 5, pp. 1027-1061, May 2007.

[23] X. He and P. Niyogi, "Locality preserving projections," in Advances in Neural Information Processing System, S. Thrun, L. Saul, and B. Schölkopf, Eds. Cambridge, MA, USA: MIT Press, 2004.

[24] C. J. C. Burges, "A tutorial on support vector machines for pattern recognition," Data Mining Knowl. Discov., vol. 2, no. 2, pp. 121-167, Jun. 1998. 
[25] J. A. Benediktsson, J. R. Sveinsson, and P. H. Swain, "Hybrid consensus theoretic classification," IEEE Trans. Geosci. Remote Sens., vol. 35, no. 4, pp. 833-843, Jul. 1997.

[26] S. Prasad and L. Mann Bruce, "Decision fusion with confidence-based weight assignment for hyperspectral target recognition," IEEE Trans. Geosci. Remote Sens., vol. 46, no. 5, pp. 1448-1456, May 2008.

[27] M. Fauvel, J. Chanussot, and J. A. Benediktsson, "Decision fusion for the classification of urban remote sensing images," IEEE Trans. Geosci. Remote Sens., vol. 44, no. 10, pp. 2828-2838, Oct. 2006.

[28] C. M. Jarque and A. K. Bera, "A test of normality of observations and regression residuals," Int. Stat. Rev., vol. 55, no. 2, pp. 163-172, Aug. 1987.

[29] O. Chapelle, V. N. Vapnik, O. Bousquet, and S. Mukherjee, "Choosing multiple parameters for support vector machines," Mach. Learn., vol. 46, no. 1-3, pp. 131-159, Jan. 2002.

[30] M. N. Do, "Fast approximation of Kullback-Leibler distance for dependence trees and hidden Markov models," IEEE Signal Process. Lett., vol. 10, no. 4, pp. 115-118, Apr. 2003.

[31] A. Villa, J. A. Benediktsson, J. Chanussot, and C. Jutten, "Hyperspectral image classification with independent component discriminant analysis," IEEE Trans. Geosci. Remote Sens., vol. 49, no. 12, pp. 4865-4876, Dec. 2011

[32] Y. Zhang, S. D. Backer, and P. Scheunders, "Noise-resistant wavelet-based Bayesian fusion of multispectral and hyperspectral images," IEEE Trans. Geosci. Remote Sens., vol. 47, no. 11, pp. 3834-3843, Nov. 2009.

[33] G. Chen and S. E. Qian, "Denoising of hyperspectral imagery using principal component analysis and wavelet shrinkage," IEEE Trans. Geosci. Remote Sens., vol. 49, no. 3, pp. 973-980, Mar. 2011

[34] J. E. Fowler and Q. Du, "Reconstructions from compressive random projections of hyperspectral imagery," in Optical Remote Sensing: Advances in Signal Processing and Exploitation Techniques, S. Prasad, L. Mann Bruce, and J. Chanussot, Eds. New York, NY, USA: Springer-Verlag, 2011, ch. 3, pp. 31-48

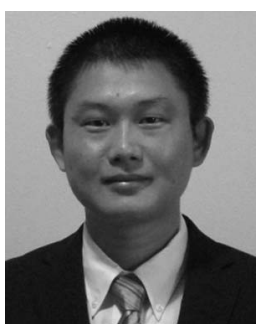

Wei Li (S'11-M'13) received the B.E. degree in telecommunications engineering from Xidian University, Xi' an, China, in 2007, the M.S. degree in information science and technology from Sun YatSen University, Guangzhou, China, in 2009, and the $\mathrm{Ph} . \mathrm{D}$. degree in electrical and computer engineering from Mississippi State University, Starkville, MS, USA, in 2012.

Subsequently, he spent one year as a Postdoctoral Researcher at the University of California, Davis, CA, USA. He is currently with the College of Information Science and Technology, Beijing University of Chemical Technology, Beijing, China. His research interests include statistical pattern recognition, hyperspectral image analysis, and data compression.

$\mathrm{Dr}$. $\mathrm{Li}$ is an active reviewer for the IEEE TRANSACTIONS ON GEOSCIENCE and Remote Sensing, the IEEE Geoscience and Remote Sensing LetTers, and the IEEE Journal of Selected Topics IN APplied EARTH OBSERVATIONS AND REMOTE SENSING.

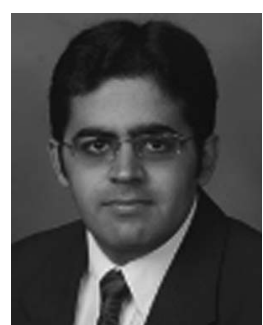

Saurabh Prasad (S'05-M'09) received the B.S. degree in electrical engineering from Jamia Millia Islamia, New Delhi, India, in 2003, the M.S. degree in electrical engineering from Old Dominion University, Norfolk, VA, USA, in 2005, and the Ph.D degree in electrical engineering from Mississippi State University, Starkville, MS, USA, in 2008.

$\mathrm{He}$ is an Assistant Professor with the Electrical and Computer Engineering Department, University of Houston (UH), Houston, TX, USA, and leads the Hyperspectral Image Analysis group at UH. His group is also affiliated with the Geosensing Systems Engineering Research Center and the National Science Foundation-funded National Center for Airborne Laser Mapping. He was the Lead Editor of the book titled Optical Remote Sensing: Advances in Signal Processing and Exploitation Techniques, published in March 2011. His research interests include statistical pattern recognition, adaptive signal processing, and kernel methods for medical imaging, optical, and synthetic aperture radar remote sensing. In particular, his current research work involves the use of information fusion techniques for designing robust statistical pattern classification algorithms for hyperspectral remote sensing systems operating under low-signal-to-noise-ratio, mixed-pixel, and small-training-sample-size conditions.

Dr. Prasad is an active Reviewer for the IEEE Transactions on Geoscience And Remote Sensing, the IEEE Geoscience and Remote Sensing LetTers, and the Elsevier Pattern Recognition Letters. He was awarded the Geosystems Research Institute Graduate Research Assistant of the Year award in May 2007 and the Office-of-Research Outstanding Graduate Student Research Award in April 2008 at Mississippi State University. In July 2008, he received the Best Student Paper Award at IEEE International Geoscience and Remote Sensing Symposium 2008 held in Boston, MA, USA. In October 2010, he received the State Pride Faculty Award at Mississippi State University for his academic and research contributions.

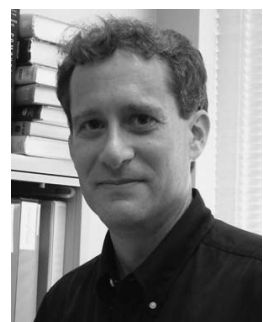

James E. Fowler (S'91-M'96-SM'02) received the B.S. degree in computer and information science engineering and the M.S. and Ph.D. degrees in electrical engineering from The Ohio State University, Columbus, OH, USA, in 1990, 1992, and 1996 , respectively.

In 1995, he was an Intern Researcher at AT\&T Labs, Holmdel, NJ, USA, and in 1997, he held a National Science Foundation-sponsored postdoctoral assignment at the Université de Nice-Sophia Antipolis, France. In 2004, he was a Visiting Professor with the Département Traitement du Signal et des Images, École Nationale Supérieure des Télécommunications, Paris, France. He is currently a Billie J. Ball Professor and the Graduate Program Director of the Department of Electrical and Computer Engineering at Mississippi State University in Starkville, MS, USA; he is also a Researcher in the Geosystems Research Institute at Mississippi State.

Dr. Fowler is an Associate Editor for the IEEE TRANSACTIONS ON IMAGE PROCESSING and the EURASIP Journal on Image and Video Processing; he formerly served as an Associate Editor for the IEEE TRANSACTIONS ON Multimedia and IEEE Signal Processing Letters. He is the Chair of the Image, Video, and Multidimensional Signal Processing Technical Committee of the IEEE Signal Processing Society and a member of the Strategic Planning Committee of the IEEE Publication Services and Products Board. $\mathrm{He}$ is the General Cochair of the 2014 IEEE International Conference on Image Processing, Paris, France, as well as the Publicity Chair of the Program Committee for the Data Compression Conference. 